\title{
Skin Deep: A Fascinating Case Report of Immunotherapy-Triggered, Treatment-Refractory Autoimmune Lichen Planus and Keratoacanthoma
}

\author{
Beatrice T. B. Preti ${ }^{a}$ b Alec Pencz ${ }^{b}$ Jeffery J. M. Cowger ${ }^{c}$ Mark \\ D. Vincent ${ }^{a, b}$ Daniel Breadner ${ }^{a, b}$ \\ aSchulich School of Medicine and Dentistry, Western University, London, ON, Canada; \\ bepartment of Medical Oncology, London Regional Cancer Program, London, ON, Canada; \\ 'Dermatology, London, ON, Canada
}

Keywords

NSCLC $\cdot$ Immunotherapy $\cdot$ Patient advocacy $\cdot$ Adverse events

\begin{abstract}
This case discusses a 62-year-old woman with de novo metastatic lung adenocarcinoma (PDL1 $>50 \%$ with a KRAS G12C mutation, ALK and EGFR negative) who was on pembrolizumab for 1 year without any significant toxicity, only low-grade dermatitis and hypothyroidism. She was transitioned to pembrolizumab every 6 weeks at $4 \mathrm{mg} / \mathrm{kg}$ and began to develop oral sores shortly thereafter. The sores proved refractory to nystatin and mouth rinses containing corticosteroids, and the patient was ultimately diagnosed with autoimmune-triggered lichen planus. Unfortunately, her symptoms also proved refractory to typical treatments for lichen planus and worsened to the point where she began to develop cutaneous lesions and difficulty swallowing. Unfortunately, she also developed a keratoacanthoma that required excision. The pembrolizumab was stopped, and the patient's symptoms improved with 5 days of systemic prednisone, metronidazole, and triamcinolone oral paste. Her NSCLC remains stable off active treatment for 6 months. This case study is on rare auto-immune toxicity as well as a keratoacanthoma from anti-PD-(L) 1 blockade, accompanied by sustained treatment response after cessation of the offending drug.
\end{abstract}




\section{Introduction}

In recent years, the arrival of immunotherapy has transformed the realm of modern oncologic management [1]. With its popularity and growing indications in cancers such as lung, melanoma, bladder, and renal cell carcinoma, the number of unusual adverse effects noted is also rising.

Lichen planus is an immune-mediated chronic inflammatory condition which causes mucocutaneous lesions. Lichen planus eruptions triggered by immunotherapy are a rare immunotherapy complication but have been previously described in the literature [2, 3]. There have also been reports of keratoacanthoma, as well as eruptive keratoacanthomas caused by immune checkpoint inhibitors [4]. To our knowledge there is a single case report of nivolumab causing both eruptive keratoacanthoma and lichen planus in a patient [5]. We herein present the first case of lichen planus with concurrent keratoacanthoma triggered by a pembrolizumab at time of a dose increase. The lichen planus was refractory to medical therapy and necessitated corticosteroids and treatment cessation.

\section{Case Presentation}

At the time of presentation to medical oncology, the patient was a 62-year-old female with a new diagnosis of de novo metastatic lung adenocarcinoma. Earlier in the month, she had presented with shortness of breath, night sweats for 6 weeks, a 10-lb weight loss over 1 year, decreased appetite, and central chest discomfort. A chest X-ray was performed, which revealed a significant pericardial effusion with significant bilateral pleural effusions. She required a pericardial drain due to hemodynamic instability. Cytology of the pericardiocentesis fluid revealed a TTF-1-positive adenocarcinoma, consistent with a lung primary.

Cross-sectional imaging revealed a $6.1 \times 6.3 \times 5.4 \mathrm{~cm}$ left upper lobe primary mass with extension into the left hilum and metastasis to a left supraclavicular node, multiple enlarged thoracic lymph nodes and pleural-based nodules, and a gluteal soft tissue deposit. The patient's prior history was remarkable for dyslipidemia and a transitional cell carcinoma of the right ureter/renal pelvis, successfully treated with BCG 18 years prior to this presentation. She was an active light smoker with a 20 pack-year smoking history.

The patient was consented for cytotoxic chemotherapy with carboplatin and pemetrexed, as treatment was needed before her pericardial effusion worsened. An urgent repeat biopsy was performed of a station 7 lymph node via EBUS for biomarker testing. The tumor was found to be strongly PD-L1 positive ( $>50 \%$ ), ALK and EGFR negative, and positive for mutated KRAS G12C and TP53. Based on this, the decision was made to pursue pembrolizumab monotherapy (a course of action also more agreeable to the patient) in lieu of the originally planned cytotoxic chemotherapy.

One week after starting pembrolizumab, the patient was readmitted to hospital for worsening shortness of breath. She underwent repeat pericardiocentesis and a pericardial window. This was tolerated well, and she continued her pembrolizumab as planned. After the second dose of pembrolizumab, the patient's subcutaneous lesion disappeared, her breathing improved, and her chest discomfort resolved. Her imaging demonstrated an ongoing partial response, with a decreased sum of the target lesion's maximum diameter of approximately $50 \%$ throughout her first year on therapy, with the only adverse effects noted of low-grade dermatitis and hypothyroidism requiring levothyroxine supplementation.

The patient continued treatment for 1 year with pembrolizumab $2 \mathrm{mg} / \mathrm{kg}$ given every 21 days. Because of the COVID-19 pandemic, she elected at that point to switch to $4 \mathrm{mg} / \mathrm{kg}$ every 


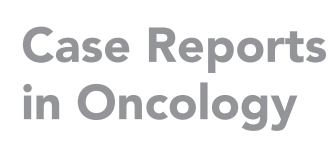

Preti et al.: Immunotherapy-Induced Treatment Refractory Lichen Planus and Keratoacanthoma
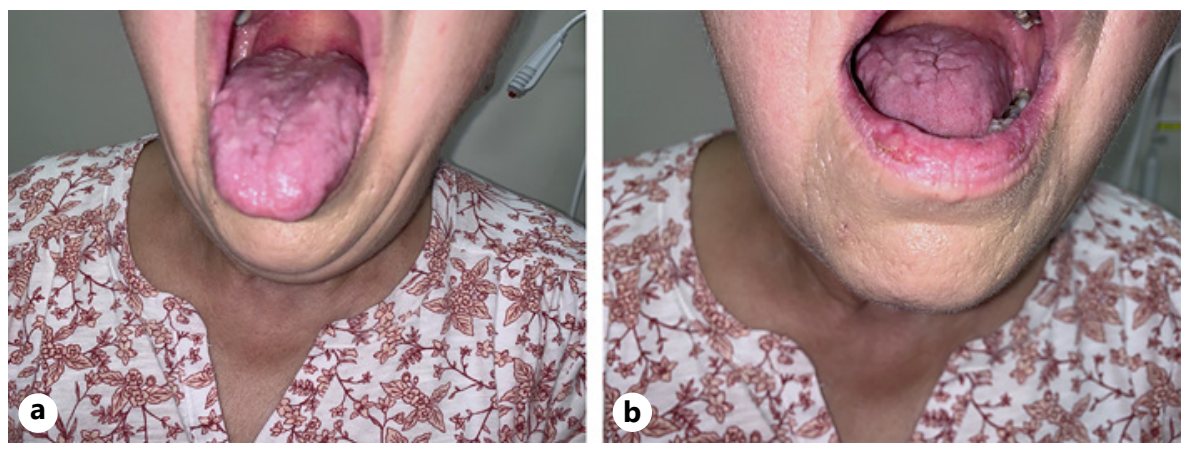

Fig. 1. Oral (a) and lip (b) sores following treatment with nystatin and negative swabs for candidiasis and herpes serology. All images are captured at the same clinic visit, prior to a third dose of pembrolizumab at $4 \mathrm{mg} / \mathrm{m}^{2}$.

42 days in order to minimize in-person contact. After 2 doses at the 42-day intervals, the patient began to develop oral sores. She was treated with nystatin for oral candidiasis, which did not resolve her symptoms. Subsequent swabs for HSV, VSV, and candida were negative. The patient continued on pembrolizumab, and her oral sores worsened. Lesions also appeared on her tongue and lips (Fig. 1). Concern was raised for autoimmune lichen planus or another autoimmune skin toxicity, and she was referred to dermatology. Imaging repeated at this time showed that her visceral disease continued to shrink.

The patient trialed Akabutu ${ }^{\circledR}$ mouthwash, a mixture of nystatin, hydrocortisone, and lidocaine. This helped alleviate symptoms initially, but her sores continued to worsen. She developed skin lesions and it became progressively more difficult for her to eat and the pembrolizumab was held.

The patient then trialed a hydrocortisone rinse, which she reports worsened her symptoms. She experienced some improvement with oral prednisone $25 \mathrm{mg} \times 6$ days, but her symptoms worsened when the steroids were discontinued. She was seen for dermatology consultation and biopsies demonstrated lichen planus as well as a keratoacnathoma (Fig. 2). She was treated with triamcinolone dental paste, clobetasol ointment, and metronidazole for the mucocutaneous lichen planus. She also required excision of the keratoacanthoma.

Currently, the patient has been off pembrolizumab for 5 months and continues to have stable disease with a further reduction in her tumor size. The oral and cutaneous lesions have largely resolved, Figure 3. The hope is that her disease will remain quiescent, with plans to rechallenge with pembrolizumab $2 \mathrm{mg} / \mathrm{kg}$ given every 21 days only when needed for progressive disease.

\section{Discussion}

This case demonstrates an unusually severe, treatment-refractory case of autoimmunemediated lichen planus, triggered by immunotherapy for metastatic lung cancer. This autoimmune phenomenon coincides with the transition to the higher dose of pembrolizumab given every 42 days. Severe skin reactions occurred at double the rate in patients receiving pembrolizumab at $10 \mathrm{mg} / \mathrm{kg}$ compared to $2 \mathrm{mg} / \mathrm{kg}$ every 21 days, but it is not possible to determine if the dose change was the cause of the lichen planus in this specific case [6]. This case highlights the importance of obtaining adequate tissue for complete biomarker testing, as this patient was preparing to start carboplatin and pemetrexed she agreed to an urgent EBUS-guided biopsy which revealed both PD-L1 >50\% IHC and a KRAS G12C mutation,

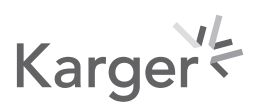




\section{Case Reports in Oncology}

\begin{tabular}{l|l}
\hline Case Rep Oncol 2021;14:1189-1193 \\
\hline DOI: 10.1159/000518313 & $\begin{array}{l}\text { ○ 2021 The Author(s). Published by S. Karger AG, Basel } \\
\text { www.karger.com/cro }\end{array}$ \\
\hline
\end{tabular}

Preti et al.: Immunotherapy-Induced Treatment Refractory Lichen Planus and Keratoacanthoma
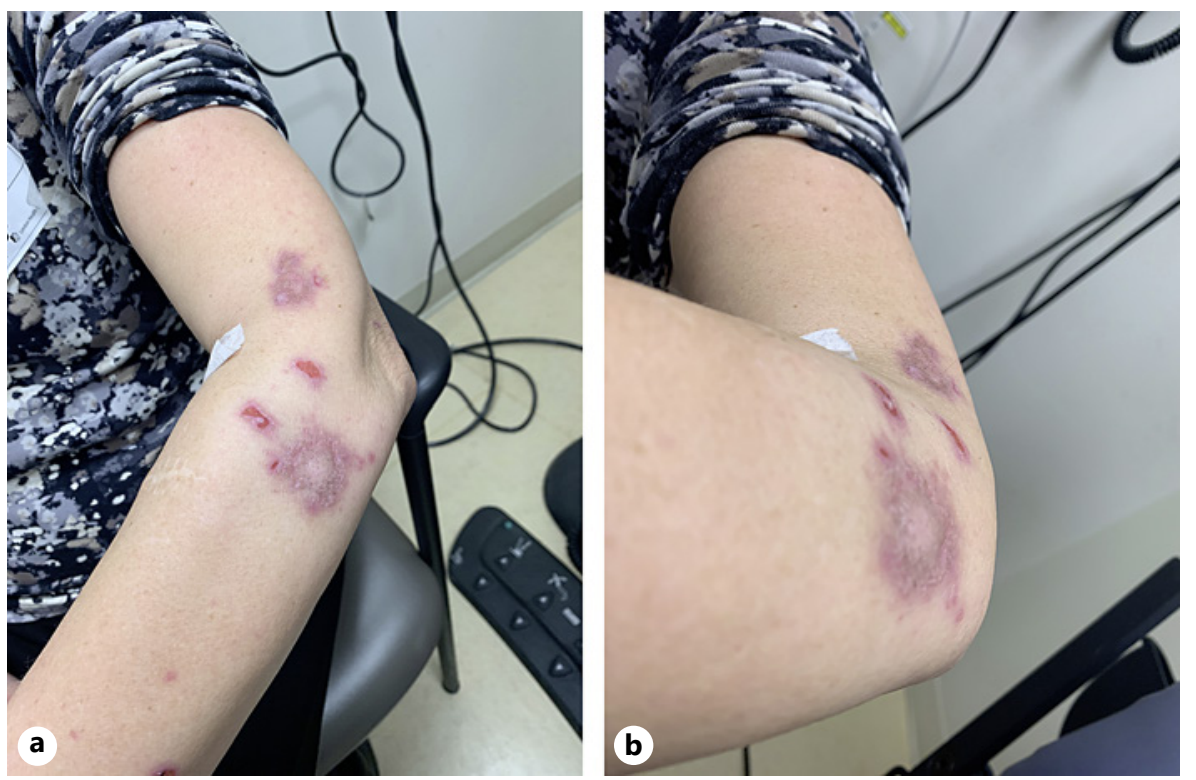

Fig. 2. Images of the left forearm (a) taken in clinic reveal the raised lesion distal (b) lesion, a keratoacanthoma, following a third dose of pembrolizumab at $4 \mathrm{mg} / \mathrm{m}^{2}$. Pembrolizumab was held at this clinic visit because of worsening quality of life.
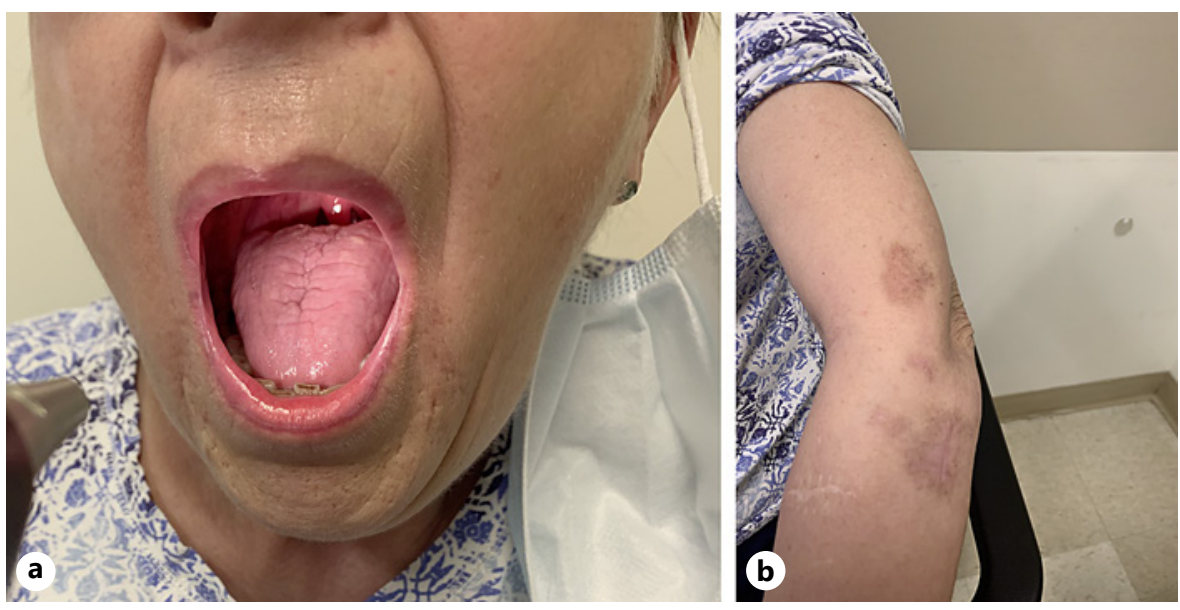

Fig. 3. Images of the (a) oral mucosa with resolution of the ulceration and (b) the left forearm with only residual scarring and pigment changes, but resolution of palpable pruritic rash.

dramatically altering her treatment options. This case also highlights the importance of a broad differential for all patients on immunotherapy, even when a cancer patient presents with a common side effect, such as mucositis. Fortunately, as in our case, the benefits of the pembrolizumab often persist beyond discontinuation of therapy.

\section{Acknowledgment}

We thank the patient in this report. She has reviewed a copy of this manuscript and supports it, hoping it will improve the experience of others. 


\section{Case Reports in Oncology}

\begin{tabular}{l|l}
\hline Case Rep Oncol 2021;14:1189-1193 \\
\hline DOI: 10.1159/000518313 & $\begin{array}{l}\text { @ 2021 The Author(s). Published by S. Karger AG, Basel } \\
\text { www.karger.com/cro }\end{array}$ \\
\hline
\end{tabular}

Preti et al.: Immunotherapy-Induced Treatment Refractory Lichen Planus and Keratoacanthoma

\section{Statement of Ethics}

This manuscript is exempt from ethical committee approval, as per institutional policy, but complies with ethical guidelines set out by the Western Research Ethics Board, at Western University, London, ON, Canada. Written informed consent was obtained from the patient for publication of this case report and the accompanying images. She has reviewed and provided feedback on this manuscript.

\section{Conflict of Interest Statement}

D.B. has served on advisory boards for Amgen Canada and Takeda. M.D.V. has served on advisory boards or received honoraria from Lilly, Roche Canada, Bristol-Myers-Squibb, Sanofi, Boehringer-Ingelheim, Amgen, Novartis, Celgene, and Merck. J.J.M.C. has served on advisory boards, received honoraria, or contributed to clinical trials for Abbvie, Amgen, Aralez Pharmaceuticals Inc., Bausch Health, Celgene, Galderma, Janssen, Johnson and Johnson, Leo Pharma Inc, Lilly, Novartis, Pfizer, Sanofi Genzyme, UCB, and Valeant. The remaining authors declare no conflicts of interest.

\section{Funding Sources}

No funding to declare.

\section{Author Contributions}

B.T.B.P. prepared the original manuscript and participated in conceptualization. A.P. edited this manuscript and participated in conceptualization. J.J.M.C. provided manuscript revisions and conceptualization. M.D.V. provided manuscript revisions. D.B. provided supervision, manuscript revisions, and conceptualization.

\section{Data Availability Statement}

All data generated or analyzed during this study are included in this article.

\section{References}

1 Kennedy LB, Salama AKS. A review of cancer immunotherapy toxicity. CA Cancer J Clin. 2020;70(2):86-104.

2 Coscarart A, Martel J, Lee MP, Wang AR. Pembrolizumab-induced pseudoepitheliomatous eruption consistent with hypertrophic lichen planus. J Cutan Pathol. 2020;47(3):275-9.

3 Yilmaz M, Mese SG, Celik U. Nivolumab-induced lichen planus. J Oncol Pharm Pract. 2020;26(3):758-60.

4 Freites-Martinez A, Kwong BY, Rieger KE, Coit DG, Colevas AD, Lacouture ME. Eruptive keratoacanthomas associated with pembrolizumab therapy. JAMA Dermatol. 2017;153(7):694-7.

5 Lee AK, Little AJ, Zubek AE. Nivolumab-induced hypertrophic lichen planus and eruptive keratoacanthomas. J Am Acad Dermatol. 2019;81(Suppl 1):AB97.

6 Herbst RS, Baas P, Kim DW, Felip E, Pérez-Gracia JL, Han JY, et al. Pembrolizumab versus docetaxel for previously treated, PD-L1-positive, advanced non-small-cell lung cancer (KEYNOTE-010): a randomised controlled trial. Lancet. 2016 Apr 9;387(10027):1540-50. 